\title{
Equilibrium Convection on a Tidally Heated and Stressed Icy Shell of Europa for a Composite Water Ice Rheology
}

\author{
Javier Ruiz
}

\begin{abstract}
Water ice I rheology is a key factor for understanding the thermal and mechanical state of the outer shell of the icy satellites. Ice flow involves several deformation mechanisms (both Newtonian and non-Newtonian), which contribute to different extents depending on the temperature, grain size, and applied stress. In this work I analyze tidally heated and stressed equilibrium convection in the ice shell of Europa by considering a composite viscosity law which includes diffusion creep, basal slip, grain boundary sliding and dislocation creep, and. The calculations take into account the effect of tidal stresses on ice flow and use grain sizes between 0.1 and $100 \mathrm{~mm}$. An Arrhenius-type relation (useful for parameterize convective models) is found then by fitting the calculated viscosity between 170 and $273 \mathrm{~K}$ to an exponential regression, which can be expressed in terms of preexponential constant and effective activation energy. I obtain convective heat flows between $\sim 40$ and $\sim 60 \mathrm{~mW} \mathrm{~m}^{-2}$, values lower than those usually deduced $\left(\sim 100 \mathrm{~mW} \mathrm{~m}^{-2}\right)$ from geological indicators of lithospheric thermal state, probably indicating heterogeneous tidal heating. On the other hand, for grain sizes larger than $\sim 0.3 \mathrm{~mm}$ the thicknesses of the ice shell and convective sublayer are $\sim 20-30 \mathrm{~km}$ and $\sim 5-20 \mathrm{~km}$ respectively, values in good agreement with the available information for Europa. So, some fundamental geophysical characteristics of the ice shell of Europa could be arising from the properties of the composite water ice rheology.
\end{abstract}

Keywerds Europa - Satellites of Jupiter - Thermal convection - Ice rheology - Heat flow · Ice shell thickness 


\section{Intreduction}

Ice rheology is a central issue for research the thermal and mechanical properties, as well as the evolution, of the icy satellites. Concretely, water ice I viscosity is decisive for the possible existence and character of convection in the outer layer of these satellites, and hence for the possible maintenance of internal oceans (e.g., Spolnn and Schubert 2002; Barr and Pappalardo 2005; Freeman et al. 2006; McKinnon 2006).

Ice flow is a complex phenomenon involving several deformation mechanisms (e.g., Duval et al. 1983; Weertman 1983; Budd and Jacka 1989; Durham and Stern 2001; Goldsby and Kohlstedt 2001), both Newtonian (such as volume and grain boundary diffusion creep) and non-Newtonian (dislocation creep, grain boundary sliding and basal slip), which contribute to different extents lepending on the temperature, grain size, and applied stress. By taking together these diverse mechanism, Goldsby and Kohlstedt (2001) have proposed a composite flow law, which should be capable of describe the flow of water ice for a wide variety of situations.

Convection in the ice shell of Europa has been investigated by numerous works, which include parameterized (e.g., Pappalardo et al. 1998; McKinnon 1999; Hussmann et al. 2002; Nimmo and Manga 2002; Ruiz and Tejero 2003; López et al. 2003; Ruiz et al. 2007) and numerical (e.g., Sotin et al. 2002; Tobie et al. 2003; Showman and Han 2004, 2005; Mitri and Showman 2005) reatments. These works, as a whole, cover a wide variety of rheological parameters, creep mechanisms, and geodynamic settings and models.

The only previous work which has analyzed convection on Europa for a composite water ice rheology was performed by Moore (2006). This author used the parameters propose for low temperature diffusion creep, grain boundary sliding, basal slip and dislocation creep, but did not take into account the effect of tidal stresses on non-Newtonian flow mechanism. However, tidal stresses are dominant for Europa (McKinnon 1999), and therefore they should have an important influence on non-Newtonian mechanisms, and hence on the total (composite) viscosity, of the water ice in Europa. In this work I analyze thermal equilibrium convection in the ice shell of Europa by considering a composite viscosity law for water ice I, but considering the effect of tidal stresses on the viscosity of non-Newtonian creep mechanisms (see McKinnon 1999).

\section{Convective Model}

Convection in the outer shell of icy satellites operates in the stagnant lid regime (e.g., McKinnon 1998; Freeman et al. 2006), in which a cold and essentially immobile lid develops above the actively convective sublayer. Although the viscosity contrast across the entire ice shell can be very large, the viscosity variation within the convective sublayer is typically of one order of magnitude [see Grasset and Parmentier (1998) and references therein]. Grasset and Parmentier (1998) have shown that convective parameterization laws derived for constant viscosity convection are applicable if the boundary conditions are properly defined. In fact, this procedure has been previously used for icy satellites (e.g., Hussmann et al. 2002, 2006; Ruiz and Tejero 2003; Multhaup and Spohn 2007; Ruiz et al. 2007). This is useful for Europa, because tidal heating is strongly temperature-depedent (-jakangas and Stevenson 1989), and it is largely restricted to the warmest ice. In these conditions, tidal heating is negligible in the stagnant lid, which can be reated separately (Hussmann et al. 2002; Ruiz and Tejero 2003). Otherwise, parameterizations for internally heated stagnant lid convection considering the same heating rate in both the stagnant lid 
and the convective sublayer (which could be more useful for the radioactively heated mantles of the terrestrial planets) are not a good analogous for the case of the ice shell of Europa, where tidal strain rates in the stagnant lid would be strongly overestimated.

Thus, here I consider a steady-state convective layer heated from within, which satisfies the relations found by Grasset and Pamentier (1998). So, the relation between the dimensionless temperature ratio and the Rayleigh number is given by

$$
\frac{k\left(T_{i}-T_{t}\right)}{H b_{c}^{2}}=a \mathrm{Ra}_{\mathrm{H}}^{\beta},
$$

where $k$ is the thermal conductivity, $T_{i}$ is the temperature of the well-mixed convective interior, $T_{t}$ is the temperature of the top of the convective layer, $H$ is the volumetric heating rate, $b_{c}$ is the thickess of the actively convective layer, $\boldsymbol{a}$ and $\boldsymbol{\beta}$ are constants (determined from numerical investigation as 2.383 and $-\mathbf{0 . 2 2 7}$ respectively; Grasset and Parmentier 1998), and $\mathrm{Ra}_{\mathrm{H}}$ is the Rayleigh number defined for an intemally heated layer,

$$
\mathrm{Ra}_{\mathrm{H}}=\frac{\alpha \rho g H b_{c}^{5}}{k \kappa \eta_{i}}
$$

where, in tum, $\alpha$ is the thermal expansion coefficient, $\rho$ is the density, $\boldsymbol{g}$ is the gravity (1.31 $\mathrm{m} \mathrm{s}^{-2}$ for Europa), $\kappa$ is the heat diffusion coefficient, and $\eta_{i}$ is the effective viscosity calculated for $T=T_{i}$. Several terms of Eqs. 1 and 2 are functions of temperature: $k=k_{\bullet} T^{-1}, \alpha=\alpha_{\bullet} T$, and $\kappa=\kappa_{\bullet} T^{-2}$, where the constants are $k_{\bullet}=621 \mathrm{~W} \mathrm{~m}^{-1}$ (Petrenko and Whitworth 1999), $\alpha_{\bullet}=6.24 \times 10^{-7} \mathrm{~K}^{-2}$ and $\kappa_{\bullet}=9.1875 \times 10^{-2} \mathrm{~m}^{2} \mathrm{~K}^{2} \mathrm{~s}^{-2}$ (Kirk and Stevenson 1987); these functions are calculated for $T=T_{i}$, since most of the convective layer is nearly isothermal. Also, ice density varies slightly with temperature and pressure (e.g., Lupo and Lewis 1979), yet adopting a constant value does not alter the results significantly; here this value is taken as $930 \mathrm{~kg} \mathrm{~m}^{-3}$. The convective heat flow can be obtained by combining Eqs. 1 and 2

$$
F_{c}=H b_{c}=\left[\frac{k\left(T_{i}-T_{t}\right) H^{1+4 \beta}}{a}\left(\frac{k \kappa \eta_{i}}{\alpha \rho g}\right)\right]^{1 /(2+5 \beta)} .
$$

In order to use isoviscous convection equations for the convective sublayer, I use (Grasset and Pamentier 1998)

$$
T_{t}=T_{i}-2.23 \frac{R T_{i}^{2}}{Q} .
$$

for adequately define the temperature at the top of the convective layer,

Equations (1-3) work if the convective layer is only heated from within, and so a lower boundary layer does not exist, but the Europa's ice shell must also be heated from below by tidal and radioactive heating in the rocky core [the radiogenic conmibution to the surface heat flow is $\sim 6-8 \mathrm{~mW} \mathrm{~m}^{-2}$ (Cassen et al. 1982; Spolm and Schubert 2002)]. However, the method here described can be used as an approximation, since the surface heat flow of Europa must be mostly generated in the warm ice of the convective sublayer (Ruiz 2005). So, here I take $T_{i}=T_{b}$, where $T_{b}$ is the temperature at the shell base, which in tum is given by the water ice melting point, as there are solid evidences for an intemal ocean on Europa (e.g., Kivelson et al. 2000), which depends on pressure $P$ as (Chizhov 1993) 


$$
T_{b}=273.16\left(1-\frac{P(\mathrm{MPa})}{395.2}\right)^{1 / 9}
$$

the pressure at the ice shell base is given by $\rho g b$, where $b$ is the total shell thickess. The stagnant lid, which is thermally conductive and heated from below, contributes to the total ice shell thickess. For a temperature-dependent thermal conductivity the thickess of the stagnant lid is

$$
b_{s l}=\frac{k_{\bullet}}{F_{c}} \ln \left(\frac{T_{t}}{T_{s}},\right.
$$

where $T_{s}$ is the surface temperature, taken as $100 \mathrm{~K}$, a value considered as representative of the mean temperature at Europa's surface (e.g., Ojakangas and Stevenson 1989).

Finally, to calculate tidal heating rates, I assume that under tidal stresses ice behave like a viscoelastic (Maxwell) material: thus, the tidal volume dissipation rate can be calculated according to (Ojakangas and Stevenson 1989)

$$
H=\frac{2 \eta \dot{\varepsilon}^{2} \mu^{2}}{\mu^{2}+\omega^{2} \eta^{2}}
$$

where $\dot{\varepsilon}$ is the strain rate, here taken as $2 \times 10^{-1 \bullet} \mathrm{s}^{-1}$, value considered as representative for the average strain rate on the icy shell of Europa (Ojakangas and Stevenson 1989; McKinnon 1999), $\mu=4 \times 10^{\circ} \mathrm{Pa}$ is the ice rigidity, and $\omega$ is the frequency of the forcing, which can be equated with Europa's mean motion, $2.05 \times 10^{-5} \mathrm{rad} \mathrm{s}^{-1}$. I take $H=H_{i}$, which somewhat overestimates tidal dissipation within the upper boundary layer (where temperatures are lower than $T_{i}$ ).

temperatures are higher than $T_{i}$ )

total tidal heating. I thus consider our tidal dissipation rate calculations to be representative for the convective layer.

I calculate ice shell stucture and heat flow by simultaneously solving Eqs. 3-7. The total ice shell thickess is taken as $b=b_{s l}+b_{c}$ in Eq. 5 .

\section{Composite Water Ice Viscosity}

The composite flow law for pure water ice I proposed by Goldsby and Kohlsted (2001) is

$$
\dot{\varepsilon}=\dot{\varepsilon}_{\text {diff }}+\left(\dot{\varepsilon}_{\text {basal }}^{-1}+\dot{\varepsilon}_{\text {gbs }}^{-1}\right)^{-1}+\dot{\varepsilon}_{\text {dist }}
$$

where $\dot{\varepsilon}$ is the total strain rate, and $\dot{\varepsilon}_{d i f f}, \dot{\varepsilon}_{\text {basal }}, \dot{\varepsilon}_{g b s}$ and $\dot{\varepsilon}_{d i s l}$ are the strain rates due, respectively, to diffusion creep, basal slip, grain boundary sliding and dislocation creep. The flow law for each individual mechanism is given by a relation of the form

$$
\dot{\varepsilon}=\frac{A \sigma^{n}}{d^{p}} \exp \left(-\frac{Q}{n R T}\right)
$$

where $A$ is the pre-exponential coefficient, $\sigma$ is the applied stress, $n$ is the stress exponent, $\boldsymbol{d}$ is the grain size, $p$ is the grain size exponent, $\boldsymbol{Q}$ is the activation energy, $R=8.31447 \mathrm{~J} \mathrm{~mol}^{-1} \mathrm{~K}^{-1}$ is the gas constant, and $T$ is the absolute temperature. For basal slip, grain boundary sliding and dislocation creep, $A$ is a constant depending on the creep mechanism; for diffusion creep, $A_{\text {diff }}=14 V_{m} D \sqrt{ }\left(R T_{)}\right.$, 
volume and $D_{v}$ is the pre-exponential volume diffusion coefficient. Diffusion creep has not been experimentally observed in water ice, although Goldsby and Kohlstedt (2001) proposed a theoretical flow law for this deformation mechanism based on the values of the constants involved.

Based upon Eq. 8 a composite viscosity law for water ice can be written as (Freeman et al. 2006)

$$
\eta=\frac{1}{\eta_{\text {diff }}^{-1}+\left(\eta_{\text {basal }}+\eta_{g b s}\right)^{-1}+\eta_{\text {dis }}^{-1}},
$$

where the subindexes have the same meaning as in Eq. 8. For diffusion creep, the viscosity is given by

$$
\eta=\frac{d^{2}}{2 A_{\text {diff }}} \exp \left(\frac{\mathbf{e}}{R T}\right)
$$

For non-Newtonian mechanisms the viscosity is given by $\eta=\sigma /(3 \dot{\varepsilon})$, where the factor 3 in the denominator is related to the axisymetric and divergent nature of the flow (e.g., Durham and Stern 2001). In the ice shell of Europa convective stresses are significantly less than fluctuating tidal stesses. McKinnon (1999) has argued that in these conditions an average effective viscosity (which can be treated as Newtonian), can be calculated for nonNewtonian mechanisms from

$$
\eta_{\text {eff }}=\eta n^{-1 / 2}=\sigma_{\text {tidat }} /\left(3 \dot{\varepsilon} n^{1 / 2}\right),
$$

where $\sigma_{\text {tidal }}$ is tidal strain.

In order to find an Arrhenius-type relation, which is useful for the parameterized convective model described in Sect. 2, I solve Eq. 10 between 170 and $273 \mathrm{~K}$ [the temperature range in the experiments performed by Goldsby and Kohlstedt (2001)], for creep parameters shown in Table 1, and then a exponential regression is found. Calculation of tidal heat dissipation rates in Sect. 2 assumes a Maxwell behavior for water ice. The strain rate for a Maxwell material is the sum of a viscous and an elastic term. The contribution of the viscous term decreases for lowering temperatures, whereas the elastic term increases. Here I assume that the ice behave totally viscous for $T=273 \mathrm{~K}$. Thus, Eqs. 8 and 10 are solved for tidal stresses satisfying $\dot{\varepsilon}=2 \times 1 \mathbf{0}^{-1 \mathbf{s}^{-1}}$ for $T=273 \mathrm{~K}$. As diffusion creep and grain boundary sliding are sensitive to the grain size, a range between 0.1 and $100 \mathrm{~mm}$ is use for this parameter. Based on polar glacial ice observations, a grain size smaller than $0.1 \mathrm{~mm}$ is unlikely (McKinnon 1999), at least if there are no impurities limiting crystal growth. On the other hand, ice grains in equilibrium with dynamic recrystallization due to

\begin{tabular}{|c|c|c|c|c|}
\hline & $A\left(\mathrm{MPa}^{-n} \mathrm{~m}^{\prime} \mathrm{s}^{-1}\right)$ & $n$ & $p$ & $Q\left(\mathrm{~kJ} \mathrm{~mol}^{-1}\right)$ \\
\hline Diffusion creep ${ }^{a}$ & $3.02 \times 10^{-8} / r$ & 1 & 2 & 59.4 \\
\hline Basal slip & $5.5 \times 10^{7}$ & 2.4 & $\bullet$ & 6 \\
\hline Grain boundary sliding & $3.9 \times 10^{-3}$ & 1.8 & 1.4 & 49 \\
\hline Dislocation creep B & $4 . \times 10^{5}$ & 4 & $\bullet$ & 60 \\
\hline
\end{tabular}
convective stresses could approach sizes of 50-100 mm (Barr and McKinnon 2006, 2007;

Table 1 Flow laws parameters for water ice (Goldsby and Kohlsted 2001)

${ }^{a}$ Flow law for diffusion creep as adjusted by Barr and Pappalardo (2005) 


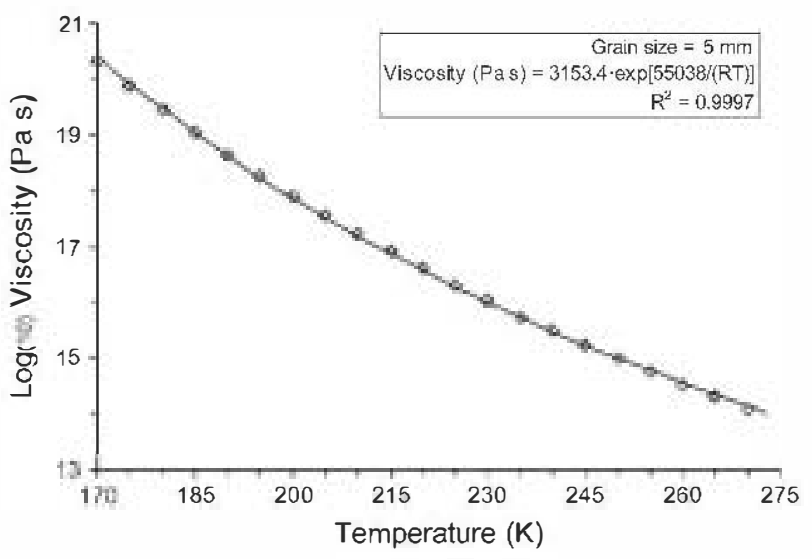

Fig. 1 Example of Arrhenius equation obtaine from an exponental regression for a grain size of $5 \mathrm{~mm}$. - pen diamonds shown viscosities obtained from (5) between 170 and $273 \mathrm{~K}$, drawn each $5^{\bullet}$ intervals in order to improve clarity. Thick solid line shows viscosities obtained from the Arrhenius equation

Tobie et al. 2006), although tidal stresses could further limit crystal growth (maybe to grain sizes close to $\sim 4 \mathrm{~mm}$; Barr and McKinnon 2006). Grain size values must be taken as averages, since this parameter could be very heterogeneous both vertically and horizontally (Tobie et al. 2006; Barr and McKinnon 2007).

The obtained exponential regression can be expressed in terms of a pre-exponential constant $B_{\mathbf{0}}$, and an effective activation energy $\boldsymbol{Q}_{\text {eff }}$, such as

$$
\eta=B_{\bullet} \exp \left(\frac{\boldsymbol{Q}_{e f f}}{R T}\right) \text {. }
$$

Figure 1 shown an example for $\boldsymbol{d}=5 \mathrm{~mm}$. The $R^{2}$ coefficient for the so-obtained exponential regressions is above 0.99 in all the cases explored. Stresses giving $\dot{\varepsilon}=$ $2 \times 10^{-1 \mathrm{~s}^{-1}}$ for $T=273 \mathrm{~K}$ are in general between $\sim 10^{4}$ and $\sim 10^{5} \mathrm{~Pa}$, in agreement with theoretical calculations of tidal stresses in the ice shell of Europa (e.g., Greenberg et al. 1998; Harada and Kurita 2006).

Figure 2 shows the value of $B_{\boldsymbol{0}}$ and $\boldsymbol{Q}_{\text {eff }}$ as functions of grain size. The curves for $B_{\boldsymbol{0}}$ and $\boldsymbol{Q}_{\text {eff }}$ have, respectively, a maximum and minimum for grain size close to $1 \mathrm{~mm}$, due to the dominant role of grain boundary sliding for this grain size. The relative importance of diffusion creep and dislocation creep increases for grain sizes lesser and greater, respectively, than $\sim 1 \mathrm{~mm}$. The implications of the properties of the composite viscosity law for convection in the Europa's ice shell are analyzed in Sect. 4.

\section{Results and Discussion}

Figure 3 shows that the convective heat flow varies between $\sim 60$ and $\sim 40 \mathrm{~mW} \mathrm{~m}^{-2}$ for grain sizes from 0.1 to $100 \mathrm{~mm}$. There is a ransition from higher to lower heat flows, which roughly coincides with the maximum and the minimum obtained, respectively, for $B_{0}$ for $\boldsymbol{Q}_{\text {eff. }}$ These heat flows are lower than the values of $\sim 100 \mathrm{~mW} \mathrm{~m}^{-2}$ usually deduced from diverse geological features used as temperature-in-depth indicators (Ruiz and Tejero 1999, 2000; Pappalardo et al. 1999; Ruiz 2005; Dombard and McKinnon 2006; 


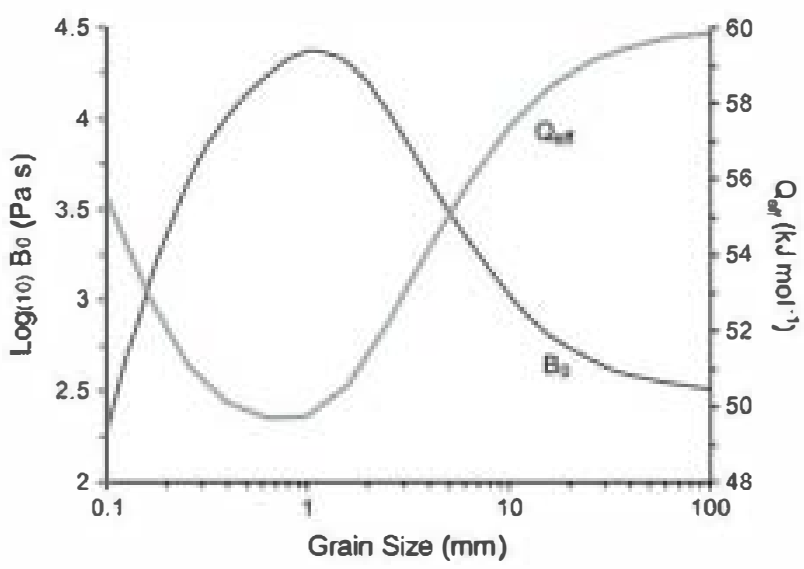

Fig. 2 Values of the pre-exponential coefficient and effective activan energy for the composite viscosity law for water ice, shown as a function of grain size

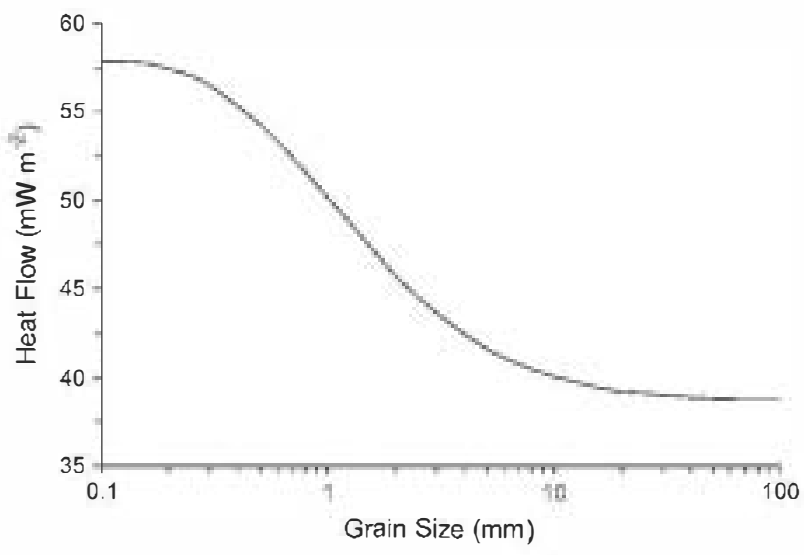

Fig. 3 Convective heat flow shows as a function of grain size

Lichtenberg et al. 2006). Previously, some works found high convective heat flows for Europa, but only for individual grain size-dependent rheologies and grain sizes lower than $1 \mathrm{~mm}$ (Nimmo and Manga 2002, Ruiz and Tejero 2003; Ruiz et al. 2007). An explanation of this discrepancy may be that high heat flows correspond to local settings with enhanced tidal dissipation. Indeed, coupled enhanced tidal heating and deformation has been previously invoked to explain both formation of double-ridges and lenticulae on Europa (e.g., Sotin et al. 2002; Nimmo and Gaidos 2002), and high heat flows (e.g., Pappalardo et al. 1999), and it has been recently shown that tidal dissipation could be much higher, even until 20-times more, in thermal plumes than in sourronding ice (Mitri and Showman 2008; Han and Showman 2010). Moreover, heat flows of 25-35 $\mathrm{mW} \mathrm{m}^{-2}$ have been proposed from the effective elastic thickess of the lithosphere supporting a plateau near the Cilix impact crater (Nimmo et al. 2003; Ruiz 2005), which suggest an heterogeneous surface heat flow on Europa. Alternatively, parameterized convective models may not be adequate to describe heat ransfer in a heterogeneously tidally heated icy shell. 


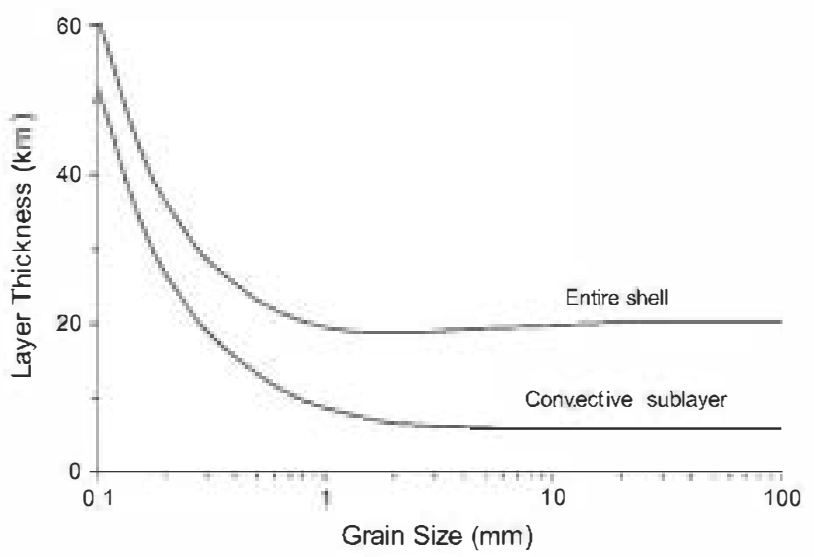

Fig. 4 Convective sublayer and total ice shell thickness as functions of grain size

Figure 4 shows the thicknesses of the actively convective sublayer and the total ice shell (given as the sum of stagnant lid and convective layer thickesses), as functions of grain size. Both thicknesses first strongly decrease, but remain roughly constants for grain sizes larger than a few millimeters. The nearly horizontal curves for larger grain sizes are due to the dominance of dislocation creep, for which $p=\boldsymbol{0}$, for these grain sizes. The ice shell is $\sim 20-30 \mathrm{~km}$ thick for grain sizes larger than $\sim 0.3 \mathrm{~mm}$, which is fairly consistent with the minimum thickess of $\sim 19-25 \mathrm{~km}$ inferred from impact craters (Schenk 2002), and with the $\sim 20 \mathrm{~km}$ of depth until an internal ocean suggested by the magnetic evidence (Schilling et al. 2004).

The origin of the areas of microchaos and features known as lenticulae (which include domes, pits and dark spots) could be related to thermal diapirs raising from a convective layer (e.g., Pappalardo et al. 1998; Nimmo and Manga 2002; Sotin et al. 2002). In this case, the convective layer would be $\sim 7-18 \mathrm{~km}$ thick (Spaun et al. 2004), roughly corresponding to the half of the observed lenticulae spacing (as proposed by Pappalardo et al. 1998). But a lower boundary layer does not exists for intemally heated convection, and hence there are no hot plumes rising (e.g., Turcotte and Schubert 2002). However, a certain amount of heat enters the ice shell from below, and tidal heating, which is strongly temperature-dependent, could therefore intensifier rising plumes (e.g., McKinnon 1999). Figure 4 shows a convective layer of $\sim 6-20 \mathrm{~km}$ for grain sizes larger than $\sim 0.6 \mathrm{~mm}$, which is in good agreement with the proposed from the lenticulae spacing.

The analysis of Moore (2006) for a composite rheology, but not considering the effects of tidal stresses on ice flow, found a shell thiclmess strongly dependent on the grain size. This is a consequence of the domination of ice flow by diffusion creep and grain boundary sliding when only convective stresses are taking into account in the calculations. However a significant role for dislocation creep arises when tidal stresses are incorporated, as expected from the high value of the stress exponent in Eq. 9 for this flow mechanism. This produces nearly constant values for the convective layer and total shell thickesses for grain sizes larger than $\sim \mathbf{0 . 3} \mathbf{m m}$. Moreover, this increased role for dislocation creep would explain that heat flows calculated for the composed rheology are lower than those obtained for grain boundary sliding in lower grain sizes (Ruiz and Tejero 2003; Ruiz et al. 2007). 


\section{Cenclusions}

The results presented in this works suggest that some fundamental geophysical characteristics of the ice shell of Europa may be a natural consequence of the properties of a composite water ice rheology coupled with tidal stresses. Indeed, the use of a composite water ice rheology, alongside the effect of tidal stresses on ice flow, for calculate equilibrium convection on Europa gives convective layer and total ice shell thiclmesses consistent, for a wide range of grain size values, with the current lmowledge about these parameters. Further application of composite rheologies to Europa and other icy worlds, as well as to different geodynamic situations, could therefore be useful for the research of the evolution and present state of the outer layers of these bodies.

Acknowledgments The author thanks Nieves López-Martínez for your discussions and encouragement, and the comments from an anonymous reviewer. The initial stages of this work were supported by a contract I3P with the CSIC co-financed from the Fondo Social Europeo (ESF), whereas the later stages were supported by a con Ramón y Cajal co-financed from the Ministerio de Ciencia e Innovación of Spain and the Fondo Social Europeo (ESF).

\section{References}

A.C. Barr, W.B. McKinnon, Convection in icy satellites with self-consistent grain size. Iunar Planet. Sci. 37, Abstract 213 (2006)

A.C. Barr, W.B. McKinnon, Convection in ice I shells and mantles with self-consistent grain size. J. Geophys. Res. 112, E 212 (2007). doi:10.1029/2006JE 12781

A.C. Barr, R.T. Pappalardo, Onset of convection in the icy Galilean satellites: influence of rheology. J. Geophys. Res. 110, E12005 (2005). doi:1 1029/2004JE 12371

W.F. Budd, T.H. Jacka, A review of ice rheology of ice sheet modelling. Cold Reg. Sci. Technol. 16, 107-144 (1989)

P.M. Cassen, S.J. Peale, R.T. Reynolds, Structure and thennal evolution of the Galilean satellites, in Satellites of Jupiter, ed. by D. Morrison (University of Arizona Press, Tucson, 1982), pp. 93-128

V.E. Chizhov, Thennodynamic properties and thennal equation of state of bigh-pressure ice phases. Prikl. Mekh. Tekh. Fiz. (Engl. Transl.) 2, 113-123 (1993)

A.J. Dombard, W.B. McKinnon, Folding of Europa's icy lithosphere: an analysis of viscous-plastic buckling and subsequent topographic relaxation. J. Swuct. Geol. 28, 2259-2269 (206)

W.B. Durham, L.A. Stern, Rheological properties of water ice-applications to satellites of the outer planets. Anmu. Rev. Earth Planet Sci. 29, 295-330 (201)

P. Duval, M.F. Ashby, I. Andennan, Rate-controlling processes in the creep of poly crystalline ice. J. Phys. Chem. 87, 4.66-4074 (1983)

J. Freeman, L. Moresi, D.A. May, Thennal convecton with a water ice I rheology: implications for icy satellite evolution. Icarus 180, 251-264 (206)

D.L. Goldsby, D.L. Koblstedt, Superplastic defonnation of ice: experimental observations. J. Geophys. Res. 106, 11, $17-1103 \bullet(201)$

O. Grasset, E.M. Pannentier, Thermal convection in a volumetrically heated, infinite Prandtl number fluid with strongly temperature-dependent viscosity: implications for planetary thermal evolution. J. Geophys. Res 103, 18,171-18181 (1998)

P. Greenberg, P. Geissler, G. Hoppa, B.R. Tufts, D.D. Durda, R. Pappalardo, J.W. Head, R. Greeley, R. Sullivan, M.H. Carr, Tectonic processes on Europa: Tidal stresses, mechanical response, and visible features. Icarus 135, 64-78 (1998)

L. Han, A.P. Showman, Couple convection and tidal dissipation in Europa's ice shell. Icanus 207, 834-844 (211)

Y. Harada, K. Kurita, The dependence of surface vidal stress on the internal structure of Europa: the possibility of cracking of the icy shell. Planet Space Sci. 54, 17-18 (206)

H. Hussmann, T. Spobn, K. Wieczerkowski, Thennal equilibrium states of Europa's ice shell: implications for internal ocean thickness and heat flow. Icarus 156, 143-151 (2002) 
H. Hussmann, F. Sohl, T. Spohn, Subsurface oceans and deep interiors of medium-sized outer planet satellites and large rans-neptunian objects. Icarus 185, 258-273 (2006)

R.L. Kirk, D.J. Stevenson, Thennal evolution of a differentated Ganymede and implications for surface features. Icarus 69, 91-134 (1987)

M.G. Kivelson, K.K. Khurana, C.T. Russell, M. Volwerk, R.J. Walker, C. Zimmer, Galileo magnetometer measurements: a stronger case for a subsurface ocean at Europa. Science 289, 1344-1343 (201)

K.A. Lichtenberg, W.B. McKinnon, A.C. Barr, Heat flux from impact ring graben on Europa. Lunar Planet. Sci. 37, Abstract 2399 (2006)

V. López, R. Tejero, J. Ruiz, Possibility of convection for diffusion (Newtonian) viscosity in the ice shell of Europa? Earth Moon Planet. 93, 281-287 (2013)

M.J. Lupo, J.S. Lewis, Mass-radius relationships in icy satellites. Icarus 40, 157-17 (1979)

W.B. McKinnon, Geodynamics of icy satellites, in Solar System Ices, ed. by B. Schmitt, C. De Bergh, M. Festou (Kluwer Academic Publishers, Dordrecht, 1998), pp. 525-55

W.B. McKinnon, Convective instability in Europa's floating ice shell. Geophys. Res. Lett. 26, 951-954 (1999)

W.B. McKinnon, On convection in ice I shells of outer solar system bodies, with detailed application to Callisto. Icarus 183, 235-250 (2006)

G. Miri, A.P. Showman, Convective-conductive sansitions and sensivity of a convecting ice shell to perturbations in heat flux and tidal-heating rate: implications for Europa. Icarus 177, 447-461 (2005)

G. Mitri, A.P. Showman, A model for the temperature-dependence of idal dissipation in convective plumes in icy satellites: implications for Europa and Enceladus. Icarus 195, 758-764 (2018)

W.B. Moore, Thermal equilibrium in Europa's shell. Icarus 180, 141-146 (206)

K. Multhaup, T. Spolm, Stagnant lid convection in the mid-sized icy satellites of Saturn. Icarus 186, $420-435$ (2007)

F. Nimmo, E. Gaidos, Causes, characteristics and consequences of convective diapirism on Europa. J. Geophys. Res. 107, doi: 10.129/2000JE01476 (2002)

F. Nimmo, N. Manga, Causes, characteristics and consequences of convective diapirism on Europa. Geophys. Res. Lett. 29, 2109 (2002). doi:1029/2002GL15754

F. Nimmo, D. J. Stevenson, Influence of early plate tectonics on the thennal evolution and magnetic field of Mars. J. Geophys. Res. 105, 11,969-11979 (2000)

F. Nimmo, B. Giese, R.T. Pappalardo, Estimates of Europa's ice shell thickness from elastically-supported topography. Geophys. Res. Lett. 30, 1233 (2003). doi:1 1029/2002GL1666

G.W. Ojakangas, D.J. Stevenson, Thennal state of an ice shell on Europa. Icarus 81, 22-241 (1989)

R.T. Pappalardo et al., Geological evidence for solid-state convection in Europa's ice shell. Nature 391, 365-368 (1998)

R.T. Pappalardo et al., Does Europa have a subsurface ocean? Evaluation of the geological evidence. J. Geophys. Res. 104, 24,15-24155 (1999)

V.F. Perenko, R.W. Whitworth, Physics of Ice (Oxford University Press, Oxford, 1999), p. 366

J. Ruiz, The heat flow of Europa. Icarus 177, 438-446 (2005)

J. Ruiz, R. Tejero, Heat flow and brittle-ducte thensition in the ice shell of Europa. Lunar Planet. Sci. Conf. 32, Abstract 1031 (1999)

J. Ruiz, R. Tejero, Heat flows through the ice lithosphere of Europa. J. Geophys. Res. 105, 23,283-23289 $(2000)$

J. Ruiz, R. Tejero, Heat flow, lenculae spacing, and possibility of convection in the ice shell of Europa. Icarus 162, 362-373 (2013)

J. Ruiz, J.A. Alvarez-Gómez, R. Tejero, N. Sánchez, Heat flow and thickness of a convective ice shell on Europa for grain size-dependent rheologies. Icarus 190, 145-154 (2007)

P.M. Schenk, Thickness constraints on the icy shells of Galilean satellites from a comparison of crater shapes. Nature 417, 419-421 (2002)

N. Schilling, K.K. Khurana, M.G. Kivelson, Limits on an inwinsic dipole moment in Europa. J. Geophys. Res. 109, E 5006 (204). doi:1 1029/2003JE 02166

A.P. Showman, L. Han, Numerical simulations of convection in Europa's ice shell: implications for surface features. J. Geophys. Res. 109, E1010 (2004). doi:10.1029/2003JE 1021

A.P. Showman, L. Han, Effects of plasticity on convection in an ice shell: implications for Europa. Icarus 177, 425-437 (2005)

C. Sotin, J.W. Head, G. Tobie, Europa: hidal heating of upwelling thennal plumes and the origin of lenticulae and chaos meling. Geophys. Res. Lett. 29, doi: 10.1029/201GL13844 (2002)

N.A. Spaun, J.W. Head, R.T. Pappalardo, Europan chaos and lenticulae: a synthesis of size, spacing, and areal density analyses. Lunar Planet. Sci. Conf. 35, Abstract 149 (204)

T. Spolm, G. Schubert, Oceans in the icy Galilean satellites? Icarus 161, 458-469 (202) 
G. Tobie, G. Choblet, C. Sotin, Tidally heated convection: constraints on Europa's ice shell thickness. J. Geophys. Res. 108, 5124 (2003). doi:101029/2003JE102099

G. Tobie, P. Duval, C. Sotin, Grain size controlling processes within Europa's ice shell. Lunar Planet. Sci. Conf. 37, Abstract 2125 (2006)

D.L. Turcotte, G. Schubert, Geodynamics: Second Edition (Cambridge University Press, Cambridge, 2002), p. 456

J. Weertman, Creep defonnation of ice. Annu. Rev. Earth Planet Sci. 11, 215-240 (1983) 